\title{
Perioperative
}

\section{management of a case of frontal glioma with idiopathic thrombocytopenic purpura}

\section{Moningi Srilata, Narmada Padhy, Dilip K Kulkarni, Sujay Parasha, Kaluvala Prasad, Kanithi Geetha, Ramachandran Gopinath}

Idiopathic thrombocytopenic purpura (ITP) is a known entity in adults and children. We describe a case who presented with frontal glioma for craniotomy and excision. On investigating, he was incidentally diagnosed to be a case of ITP. Although the patient did not have any symptoms, his platelet count was found to be low. The risk of intra-operative and post-operative bleeding in patients with ITP is increased, especially in patients undergoing craniotomy with low platelet counts. Peri-operative medical and replacement management with remedial measures is required for a successful outcome in patients undergoing craniotomy with ITP.
Chronic idiopathic thrombocytopenia in adults is usually asymptomatic with an insidious onset. Although death is rare, the usual causes of death from idiopathic thrombocytopenic purpura (ITP) is usually life-threatening bleeding, most commonly being intracranial haemorrhage. ${ }^{[1]}$ They are usually refractory to therapy. Preoperative identification and optimisation of these patients with significantly low platelet counts is a peri-operative challenge for the anaesthesiologist, especially for patients undergoing intracranial surgeries or any major surgery.

A 50-year-old male patient was diagnosed with frontal astrocytoma. His presenting features included focal seizures involving the left upper and lower limbs with generalisation. There was neither any history of bleeding manifestations, petechie or ecchymoses nor any history of blood transfusions. He had past history of jaundice which was managed conservatively.

The routine preoperative examination of peripheral blood smear showed neutrophilic leucocytosis with severe thrombocytopenia with a platelet count of $20 \times 10^{9} /$ L. His coagulation parameters were normal. He was further evaluated for thrombocytopenia. The bone marrow aspirate revealed increased megakaryocytes with hypolobate forms suggestive of ITP. Ultrasonography of the abdomen showed hepatomegaly with fatty changes. Anti-nuclear and anti-cardiolipin antibodies were tested negative. A diagnosis of chronic stable ITP was made. 
A haematologist consultation was sought to treat thrombocytopenia as the frontal glioma that required early surgery. He was initially treated with 4 units of platelet rich plasma transfusion. The improvement in platelet count was very transient. He was, therefore, started on injection methyl prednisolone $1 \mathrm{~g}$ daily for 5 days for a quicker response. Slowly the platelet counts improved. He was then shifted to oral prednisolone $20 \mathrm{mg}$ twice daily with incremental increase to $30 \mathrm{mg}$ twice daily. The counts were never persistently normal even for 2-3 days. He was started on i.v. immunoglobulins $400 \mathrm{mg} / \mathrm{kg} /$ day by infusion given over 1 hour for 4 days with good response and consistent improvement in the platelet counts. Finally after 34 days of admission, platelet counts were stabilised and the patient was taken up for craniotomy and excision of the glioma. The peri-operative trend of platelet counts was as shown in Figure 1.

Standard anaesthesia management was instituted as per institutional protocol. Monitoring included heart rate, electrocardiogram, end tidal gases, arterial blood pressure, central venous pressure, volume status, temperature and blood loss. Injection methyl prednisolone $1 \mathrm{~g}$ was given over 20 minutes before induction as a prophylactic measure. Laryngoscopy and intubation was performed with great care to avoid mucosal bleeding. The intra-operative period was uneventful with minimal blood loss. The patient was conscious, oriented and responding to commands. There were no fresh post-operative neurological deficits noted. The post-operative bleeding in the drain was minimal. In the post-operative period, he was started on i.v. dexamethasone $4 \mathrm{mg}$ four times daily. The immediate post-operative platelet count was $130 \times 10^{9} / \mathrm{L}$. And on the next day, he was shifted to oral tab prednisolone $30 \mathrm{mg}$ twice daily which was then tapered every week with intermittent measurement of platelet counts. Histopathological examination of the excised tumour confirmed fibrillary astrocytoma (WHO grade-II). He

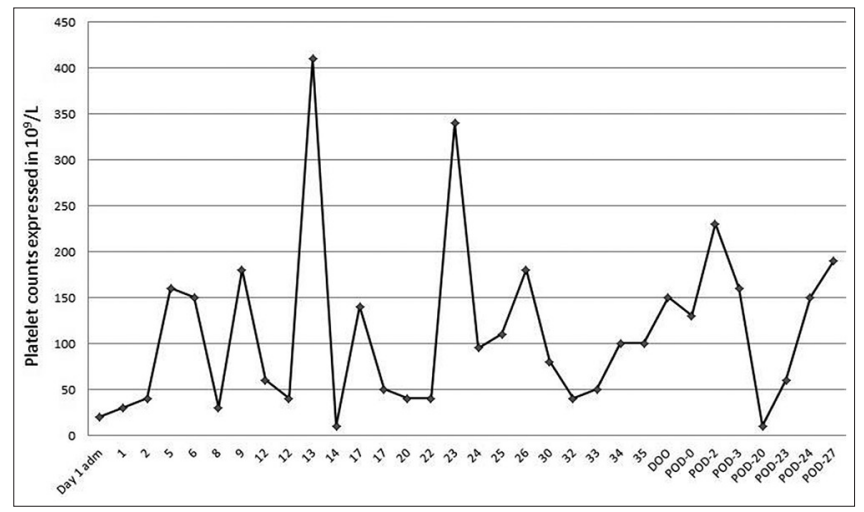

Figure 1: Trend of change in the platelet counts with time and treatment. Abbreviations: Adm. - Admission, DOO - Day of operation, POD - Post-operative day was then advised for post-surgery radiotherapy for further management.

ITP primarily is a disorder of increased platelet destruction, probably caused by the development of autoantibodies to platelet-membrane antigens. In about one-third of adults with ITP, the condition is persistent and relatively resistant to most treatments. The data on the natural history of untreated disease in adult patients with thrombocytopenia is lacking. Patients with ITP with very low platelet counts have an increased risk for potentially life-threatening bleeding, most commonly being intracranial haemorrhage. ${ }^{[1]}$ Pre-operative stabilisation of platelet counts at least to $100 \times 10^{9} / \mathrm{L}$ is a requisite for major intracranial surgery. Although the guidelines for management advocate steroid therapy, it requires about 2-3 weeks for the response. As the patient required early improvement to accomplish the surgery, platelet transfusions were administered to normalise the counts. But the patient exhibited only transient improvement possibly due to destruction of the transfused platelets by the autoantibodies. The response to steroids was also not adequate to proceed for surgery. ${ }^{[2]}$ The addition of immunoglobulins to the therapy enhanced the response. Combination of immunoglobulins and steroids are effective not only in reducing the infusion reactions and aseptic meningitis but also enhances intravenous immunoglobulin response. ${ }^{[2]}$ Older patients have haemorrhagic complications more often than do younger patients with the same platelet counts. ${ }^{[3]}$ Lotan et al., have documented a case report of intracranial haemorrhage simulating brain tumour in a 6-year-old male with ITP. ${ }^{[4]}$ Similar cases were reported in adults too. ${ }^{[5,6]}$

This patient had asymptomatic chronic ITP with very low platelet. The safe platelet count in these patients to undergo intracranial surgery is not specifically reported, though generally platelets count of atleast $>100 \times 10^{9} / \mathrm{L}$ is recommended. Patients who are refractory to therapy have difficulties in achieving these levels and even if attained, are difficult to sustain due to the presence of autoantibodies. After a thorough Medline search, no such case of an intracranial glial tumour with ITP as managed by us was reported. The patient did not exhibit any bleeding tendency or bleeding into the tumour even at the time of low platelet counts. We waited for nearly a month to optimise the platelet count and obtain a consistent improvement in the platelet count as the possibility of developing low platelet counts and post-operative intracranial bleeding with refractory ITP were high. ${ }^{[2]}$ Steroids were continued intra and post-operatively. The post-opertative platelet counts were maintained at more than $100 \times 10^{9}$. There was no excessive intraoperative bleeding or post-operative neurological deficit. Treatment with 
immunoglobulin therapy and anti-Rh-D therapy would be useful in chronic patients not responding to steroids. If the medical management fails to improve the platelet count, other options such as splenectomy, immune-suppressants, corticosteroid sparing agents, monoclonal antibodies and thrombopoietin receptor agonists would be beneficial. ${ }^{[2]}$

A routine preoperative platelet count was considered for this patient though he was asymptomatic in the pre-operative period. ${ }^{[7]}$ This helped in identification of chronic ITP and optimises the patient adequately and thereby avoids potentially catastrophic complication of peri-operative intracranial bleeding during craniotomy.

\section{REFERENCES}

1. British Committee for Standards in Haematology General Haematology Task Force. Guidelines for the investigation and management of idiopathic thrombocytopenic purpura in adults, children and in pregnancy. $\mathrm{Br} \mathrm{J}$ Haematol 2003;120:574-96.

2. Provan D, Stasi R, Newland AC, Blanchette VS, Bolton-Maggs P, Bussel JB, et al. International consensus report on the investigation and management of primary immune thrombocytopenia. Blood 2010;115:168-86.

3. Cohen YC, Djulbegovic B, Shamai-Lubovitz O, Mozes B.
The bleeding risk and natural history of idiopathic thrombocytopenic purpura in patients with persistent low platelet counts. Arch Intern Med 2000;160:1630-8.

4. Lotan CS, Wald U, Ludomirsky A, Cividalli G. Intracranial hemorrhage simulating brain tumor in immune thrombocytopenic purpura. Eur J Pediatr 1983;141:127-8.

5. Ramani A, Raja A, Ravi TS, Kundaje GN, Rao RM, Rao PN. Idiopathic thrombocytopenic purpura mimicking intracranial tumour. J Indian Med Assoc 1989;87:239-40.

6. Awerbuch G, Sandyk R. Intracranial haemorrhage in a 26 year-old woman with idiopathic thrombocytopenic purpura. Postgrad Med J 1987;63:781-3.

7. National Collaborating Centre for Acute Care (UK). Preoperative Tests: The Use of Routine Preoperative Tests for Elective Surgery. London: National Collaborating Centre for Acute Care (UK); 2003 Jun. (NICE Clinical Guidelines, No. 3) 6, Guideline for Preoperative Investigations in Patients Undergoing Elective Surgery. Available from: http:/www.ncbi.nlm.nih.gov/books/ NBK48482/[Last accessed on 2014 Mar 26].

\begin{tabular}{|l|l|}
\hline \multicolumn{2}{|c|}{ Access this article online } \\
\hline Quick Response Code: & Website: \\
\hline & www.jnaccjournal.org \\
\hline
\end{tabular}

